\title{
The Communicable Disease Surveillance System in the Kosovar refugee camps in the former Yugoslav Republic of Macedonia April-August 1999
}

Sergio Brusin

The crisis in Kosovo in spring 1999 has resulted in a major exodus of people fleeing the former autonomous province of Serbia. The major influxes of refugees headed toward Albania and the former Yugoslav Republic of Macedonia (fYROM). The total number of Kosovar refugees that entered the fYROM in spring 1999 has been estimated by UNHCR at more than 270000 . The total population of the fYROM is little more than 2200000 inhabitants, and before the crisis its National Health System was already stretched to the limit trying to provide free health care to its citizens.

In fYROM the vast majority of refugees have found refuge in the north west part of the country where there is a substantial proportion of ethnic Albanians. The refugees have been hosted in camps and by local families.

At the height of the crisis the number of refugees in camps was in excess of 120000 in seven destination camps plus a transit camp at the Blace border between Kosovo and fYROM. Because of the continuous influx into the country and the humanitarian and medical evacuation programmes, the total number of refugees that transited trough camps in fYROM from April to July 1999 is much higher with estimates nearing 200000.

UNHCR provided daily reliable population estimates both for the refugees in camps and in host families, but because of the logistical difficulties of a registration process the exact numbers cannot be calculated.

The seven camps were built in successive steps, starting in April 1999, trying to respond to the increasing number of refugees. The camps were managed by UNHCR, which coordinated the work of the International Organisations (IOs), National Agencies (NAs) and the Non Governmental Organisations (NGOs) that provided, directly or indirectly, health care, shelter, food, sanitation, clothing, etc, to the refugees.

Although some information was available on the health status of Kosovars before they were forced to leave Kosovo, there was little systematic information available on their current health status since arriving in fYROM. It was known that some infectious diseases were an important concern in Kosovo before the humanitarian crisis, and the effects of displacement on the refugees (living rough for days and then the difficult conditions in the camps in the beginning) were an additional preoccupation.

It is well known how easily communicable diseases can spread in a camp situation, even with the best hygienic measures implemented.
The need for a surveillance system that allowed early detection and response to possible outbreaks was essential.

The setting up of a Communicable Disease surveillance system also gave the opportunity to use the same information network to collect data on the general health of the camp population, on some indicators to use in the provision of health services and in some proxy for crowding and access to water.

\section{Objectives of the system}

The surveillance system in the Kosovar refugee camps in fYROM had one primary objective and various secondary ones. The primary objective was:

- early detection of communicable disease events of epidemic or life threatening potential in order to respond quickly with prevention and control measures.

The secondary objectives were:

- obtaining information on the trends of major infectious diseases in order to direct prevention policies;

- collect some indicators on the general health of the camp population;

- collect some indicators on the reproductive health of the camp population;

- collect some information on the provision of health care in the camps;

- provide feedback to each reporting agency on the situation in its camp;

- provide information to media and the public on the communicable disease situation in the camps.

Obviously, given the less than ideal situation in fYROM this spring, not all of the objectives could always be achieved.

\section{Logistic situation}

The seven main refugee camps were situated in north west fYROM, in two wide valleys through which the river Vardar flows. In the valley where the cities of Gostivar and Tetovo are, there were four camps. The other three camps were situated in the valley were Skopje stands. The distance between Skopje, where the headquarters of all the major organisations were, and the furthest camp was about $80 \mathrm{~km}$, or one and a half hours by car. Telephones worked reasonably well, and there was an FM radio network maintained by UNHCR. Hospitals in Gostivar and Tetovo had a microbiology laboratory where some bacteriology could be performed, and the University Hospital in Skopje had more sophisticated facilities. Hospital laboratories were available to receive 
WEEKLY HEALTH REPORT IN REFUGEE CAMPS IN fYROM

\begin{tabular}{|c|c|c|c|}
\hline NAME OF CAMP: & \multicolumn{3}{|c|}{ HEALTH PROVIDER: } \\
\hline DATE*: & \multicolumn{3}{|c|}{ REPORTED BY: } \\
\hline \multicolumn{4}{|c|}{ *Put the date of the last day of the week. Weeks start on Fridays and end on Thursdays } \\
\hline \multirow{2}{*}{$\begin{array}{c}\text { COMMUNICABLE } \\
\text { DISEASES/SYNDROMES }\end{array}$} & \multicolumn{3}{|c|}{ NEW CASES } \\
\hline & $\begin{array}{l}\text { Less than } 5 \\
\text { years old }\end{array}$ & $\begin{array}{l}5 \text { years old or } \\
\text { more }\end{array}$ & Total \\
\hline \multicolumn{4}{|l|}{ Bloody diarrhoea* } \\
\hline \multicolumn{4}{|l|}{ Watery diarrhoea } \\
\hline \multicolumn{4}{|l|}{ Suspected cholera* } \\
\hline \multicolumn{4}{|l|}{ Upper respiratory infections } \\
\hline \multicolumn{4}{|l|}{ Pneumonia } \\
\hline \multicolumn{4}{|l|}{ Measles* } \\
\hline \multicolumn{4}{|l|}{ Suspected meningitis* } \\
\hline \multicolumn{4}{|l|}{ Acute jaundice syndrome* } \\
\hline \multicolumn{4}{|l|}{ Sexually transmitted diseases } \\
\hline \multicolumn{4}{|l|}{ Scabies } \\
\hline \multicolumn{4}{|l|}{ Head lice } \\
\hline \multicolumn{4}{|l|}{ Acute flaccid paralysis\# } \\
\hline \multicolumn{4}{|l|}{ Fever of unknown origin (FUO) } \\
\hline Special Conditions & & NEW CASES & \\
\hline Trauma/Injury & & & \\
\hline
\end{tabular}

*Diseases with epidemic potential - report immediately to health co-ordinator - follow the protocols for microbiological or serological confirmation already established.

\# Any case of AFP must be referred to a State Hospital for the usual confirmation procedure.

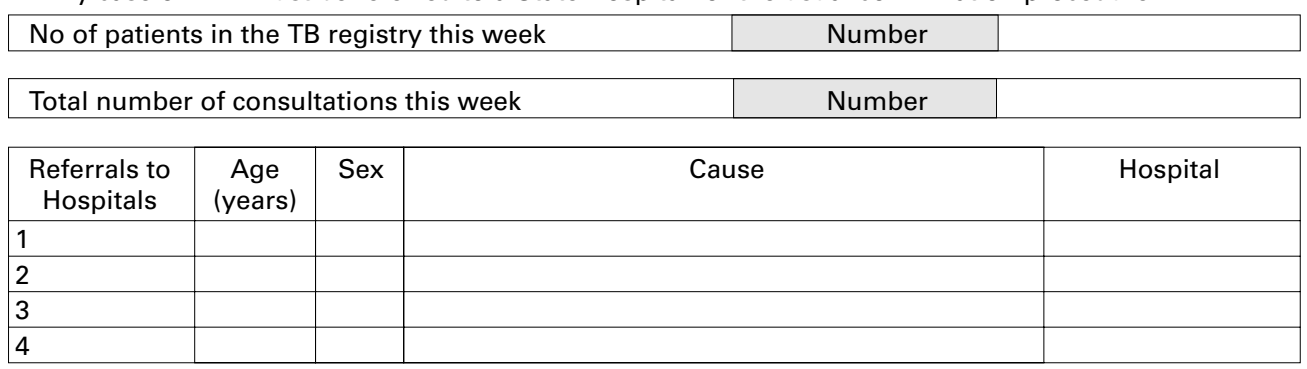

\begin{tabular}{|l|l|l|l|}
\hline $\begin{array}{l}\text { No of } \\
\text { deaths }\end{array}$ & $\begin{array}{c}\text { Age\# } \\
\text { (years) }\end{array}$ & Sex & Main cause of death \\
\hline 1 & & & \\
\hline 2 & & & \\
\hline 3 & & & \\
\hline 4 & & & \\
\hline
\end{tabular}

\# Specify in case of a neonatal dead (less than 1 month old) or an infant dead (less than 1 year old)

\begin{tabular}{|l|l|}
\hline & \multicolumn{1}{|c|}{ Number } \\
\hline Births & \\
\hline Still-born & \\
\hline
\end{tabular}

W.H.O. should receive the forms by Friday evening at the latest to include the data in the Tuesday issue of the Health Bulletin.

Contacts: Tel 386-075/76, Fax 386-063.

Figure 1 Weekly report form.

specimens at short notice and perform bacteriological tests on an emergency basis reasonably quickly. In June, hospitals inside the camps had the capability to perform some microbiological tests on their own.

In the bigger camps (Cegrane, Stankovec and Brazda) health care was provided by various agencies that sometimes worked as secondary care facilities accepting referrals. In the smaller camps (Neprosteno, Senekos, Radusha and Bojane) health care was provided by single agencies and referrals were directed to local hospitals or, seldomly, to other camps.
Twice a week all health agencies working with refugees met in Skopje in a health coordination meeting.

\section{Data collection}

A two tier system has been built according to the urgency of the reporting.

Cases of bloody diarrhoea, suspected cholera, suspected measles, suspected meningitis, acute jaundice syndrome and acute flaccid paralysis had to be reported immediately by telephone or radio to the WHO office in Skopje 
- Each Agency providing Health Care in the camps only needs to fill in a form per week.

- The data in the form should be the summary of the reports of cases seen in the week.

- Weeks start on Fridays and end on Thursdays to allow us to provide on Tuesdays the tables attached to the Health Bulletin.

- OPDs and Agencies providing Primary Health Care should report all new cases seen.

- Hospitals (G-RC and N-RC) should provide only data on people seen in the emergency department. Do not report referred cases.

- Trauma/injury is added to the new cases to be reported.

- Cases of Communicable Disease or Trauma/injury should be reported divided in two categories "Less than 5 years old" and "5 or more years old".

- AFP (Acute Flaccid Paralysis) has been introduced in the forms. We would like to remind you that each case must be immediately referred to a Macedonian hospital to follow the usual confirmation procedure.

- The "Total number of consultations" should include all consultations provided by the Agency in the reporting week. It is not the sum of the cases reported above.

- Tuberculosis is subject to a different surveillance. All agencies accredited for TB treatment should send weekly a copy of the "TB Register 03" to W.H.O.

- List all the referral to Hospitals (both State Hospitals or Camp Hospitals) with the cause and the receiving Hospital.

- The number of deaths should be reported with age, sex and cause of death. Please specify besides the age if it is a neonatal death (infants less than 1 month old) or an infant death (child less than 1 year old).

- The number of deaths include the refugees that die in the camps and the deaths in referred hospital or accidents outside the camp when the information is available.

- Please report the number of births and the number of still-born. The numbers should include all deliveries of women living in the camp irrespective of the place of delivery (e.g. if a mother is referred to a state hospital for delivery the birth should be registered as a birth in the camp).

- Microbiological or serological investigation are required for Bloody Diarrhoea, Suspected Cholera, Suspected Measles, Suspected Meningitis and Acute Jaundice Syndrome. Please follow the already established protocols with the Public Health Institutes.

- In case of doubts, difficulties or clarifications please contact the W.H.O. Humanitarian Assistance Office in Skopje Tel: 091386075 or 091386076 , Fax: 091386063

The report forms, the case definitions and this leaflet will be provided in English, Albanian and Macedonian. Local translations of the case definitions and this instruction leaflet are welcomed, but it is advisable to discuss them with WHO staff.

Figure 2 Instruction sheet.

and to the Republic Institute for Health Protection (RIHP). Specimens had to be immediately collected and sent by car to the appropriate laboratory for confirmation.

Written protocols were distributed to all health care facilities in the camps with case definitions, specimen collection and transport guidelines, details of the closest laboratory, telephone numbers and contact persons. Laboratories fed back to the specimen sender, the WHO office and the RIHP.

In addition to this system a weekly reporting form had to be filled in by all health facilities providing primary health care or emergency services in each camp. To avoid duplications secondary health care facilities did not report (fig 1).

Each reporting facility received, together with the forms, a case definition sheet and a copy of the transmission protocols with relevant telephone numbers and contact persons. On the back of each form there was a set of instructions on how to fill in the form and when and to whom to deliver it (fig 2).

All forms, case definitions, protocols and information sheets were distributed to all reporting agencies in English, Albanian and Macedonian.

WHO teams regularly travelled to all camps and to all health facilities to ensure the provision of the right forms, instruct the reporting persons, assure the flow of information and collect the forms if necessary.

All agencies were expected to provide the most recent completed surveillance form each week at the Friday Health Co-ordination Meeting in Skopje to allow WHO to publish the Weekly Health Bulletin with all the 
Table 1 Example of the CD summary weekly table in the Health Bulletins

\begin{tabular}{|c|c|c|c|c|c|c|c|c|c|c|c|c|}
\hline \multicolumn{7}{|c|}{ New cases of communicable diseases reported from camps in fYROM } & \multicolumn{5}{|c|}{5 fune-11 fune 1999} & \\
\hline Camp & \multicolumn{3}{|c|}{ Brazda } & \multicolumn{3}{|c|}{ Stankovec II } & \multicolumn{3}{|c|}{ Radusha } & \multicolumn{3}{|c|}{ Bojane } \\
\hline Week & \multirow{2}{*}{\multicolumn{3}{|c|}{ 5-11 June }} & \multicolumn{3}{|c|}{ 5-11 June } & \multicolumn{3}{|c|}{ 5-11 June } & \multicolumn{3}{|c|}{ 5-11 June } \\
\hline \multirow{2}{*}{ Communicable diseases/syndromes } & & & & New & & & New & & & New & & \\
\hline & $<5$ & $>5$ & Total & $<5$ & $>5$ & Total & $<5$ & $>5$ & Total & $<5$ & $>5$ & Total \\
\hline Bloody diarrhoea* & 1 & 1 & 2 & 0 & 0 & 0 & 0 & 0 & 0 & 0 & 0 & 0 \\
\hline Watery diarrhoea & 75 & 42 & 117 & 6 & 6 & 12 & 22 & 15 & 37 & 11 & 16 & 27 \\
\hline Suspected cholera ${ }^{\star}$ & 0 & 0 & 0 & 0 & 0 & 0 & 0 & 0 & 0 & 0 & 0 & 0 \\
\hline Upper respiratory infections & 182 & 233 & 415 & 13 & 31 & 44 & 45 & 81 & 126 & 55 & 78 & 133 \\
\hline Pneumonia & 5 & 8 & 13 & 0 & 0 & 0 & 5 & 6 & 11 & 4 & 6 & 10 \\
\hline Measles $\star$ & 0 & 0 & 0 & 0 & 0 & 0 & 0 & 0 & 0 & 0 & 0 & 0 \\
\hline Suspected meningitis* & 0 & 0 & 0 & 0 & 0 & 0 & 0 & 0 & 0 & 0 & 0 & 0 \\
\hline Acute jaundice syndrome ${ }^{\star}$ & 0 & 0 & 0 & 0 & 0 & 0 & 0 & 0 & 0 & 0 & 0 & 0 \\
\hline Sexually transmitted diseases & 0 & 0 & 0 & 0 & 0 & 0 & 0 & 6 & 6 & 0 & 0 & 0 \\
\hline Scabies/Head lice & 6 & 12 & 18 & 0 & 6 & 6 & 0 & 1 & 1 & 7 & 12 & 19 \\
\hline Acute flaccid paralysis $\star$ & 0 & 0 & 0 & 0 & 0 & 0 & 0 & 0 & 0 & 0 & 0 & 0 \\
\hline Fever of unknown origin (FUO) & 18 & 14 & 32 & 0 & 0 & 0 & 4 & 10 & 14 & 22 & 12 & 24 \\
\hline Trauma/injury & 5 & 1 & 6 & 4 & 8 & 12 & 0 & 0 & 0 & 6 & 5 & 11 \\
\hline Total & 292 & 311 & 603 & 23 & 51 & 74 & 76 & 119 & 195 & 105 & 129 & 224 \\
\hline Estimated population $\star \star$ & 210 & & & 2045 & & & 2500 & & & 3971 & & \\
\hline Camp & \multicolumn{3}{|c|}{ Neprosteno } & \multicolumn{3}{|c|}{ Senekos } & \multicolumn{3}{|c|}{ Cegrane } & & & \\
\hline Week & \multicolumn{3}{|c|}{ 5-11 June } & \multicolumn{3}{|c|}{ 5-11 June } & \multicolumn{3}{|c|}{ 5-11 June } & & & \\
\hline \multirow{2}{*}{ Communicable diseases/syndromes } & \multicolumn{3}{|c|}{ New cases } & \multicolumn{3}{|c|}{ New cases } & \multicolumn{3}{|c|}{ New cases } & & & \\
\hline & $<5$ & $>5$ & Total & $<5$ & $>5$ & Total & $<5$ & $>5$ & Total & & & \\
\hline Bloody diarrhoea* & 0 & 0 & 0 & 0 & 0 & 0 & 0 & 2 & 2 & & & \\
\hline Watery diarrhoea & 20 & 35 & 55 & 56 & 56 & 112 & 79 & 100 & 179 & & & \\
\hline Suspected cholera ${ }^{\star}$ & 0 & 0 & 0 & 0 & 0 & 0 & 0 & 0 & 0 & & & \\
\hline Upper respiratory infections & 31 & 63 & 94 & 63 & 106 & 169 & 184 & 242 & 426 & & & \\
\hline Pneumonia & 0 & 0 & 0 & 3 & 4 & 7 & 0 & 2 & 2 & & & \\
\hline Measles * & 0 & 0 & 0 & 0 & 0 & 0 & 1 & 0 & 1 & & & \\
\hline Suspected meningitis* & 0 & 0 & 0 & 0 & 0 & 0 & 0 & 0 & 0 & & & \\
\hline Acute jaundice syndrome ${ }^{\star}$ & 0 & 0 & 0 & 0 & 0 & 0 & 0 & 0 & 0 & & & \\
\hline Sexually transmitted diseases & 0 & 0 & 0 & 0 & 0 & 0 & 0 & 0 & 0 & & & \\
\hline Scabies/Head lice & 4 & 19 & 23 & 0 & 2 & 2 & 2 & 319 & 321 & & & \\
\hline Acute flaccid paralysis $\star$ & 0 & 0 & 0 & 0 & 0 & 0 & 0 & 0 & 0 & & & \\
\hline Fever of unknown origin (FUO) & 0 & 0 & 0 & 3 & 2 & 5 & 19 & 15 & 34 & & & \\
\hline Trauma/injury & 0 & 4 & 4 & 0 & 3 & 3 & 4 & 1 & 5 & & & \\
\hline Total & 55 & 121 & 176 & 125 & 173 & 298 & 289 & 681 & 970 & & & \\
\hline Estimated population ${ }^{\star \star}$ & 8271 & & & 6857 & & & 408 & & & & & \\
\hline
\end{tabular}

${ }^{\star}$ Diseases with epidemic potential.

${ }^{\star} \star$ Weekly average on daily UNHCR data.

feedback tables and graphs. To coordinate reporting, Health Co-ordination Meetings and production of the Weekly Health Bulletin, all the participants agreed to start the weeks on Friday and to end them on Thursday.

\section{Feedback and Health Bulletins}

Feedback, from the laboratories or from WHO, on the diseases of epidemic potential to be reported immediately, was provided to the reporting agency and to all health agencies in the same camp as soon as the information was available. At the same time WHO informed UNHCR health personnel on an informal basis and RIHP on a formal one.

All other data were collected, organised in tables, graphs and free text if necessary and provided with the Weekly Health Bulletin to all health agencies working with refugees in fYROM, all IOs, the Macedonian Ministry of
Health, the WHO offices in the region, WHOEURO and WHO-Headquarters. The bulletin was distributed both in hard copies and in email format.

Data were organised in tables summarising the weekly reporting of each camp.

Trends in the past six weeks of reporting were given for each camp with the rates per 1000 for selected diseases (table 2 and fig 3). With the observed rates a range equal to the double of the standard deviation of the rate per 1000 in the past six weeks was displayed to facilitate detection of unexpected increases (possible outbreaks) or decreases (possible sign of system breakdown).

All other data were summarised in customised paragraphs in the Weekly Health Bulletin, mostly in text style. Deaths and births were provided as crude numbers and not as rates because of the difficulty of calculating a

Table 2 Example of the table of the rates of reporting per each camp

\begin{tabular}{|c|c|c|c|c|c|c|c|c|c|c|c|}
\hline \multicolumn{8}{|c|}{ Camp Brazda Communicable Diseases report rates per 1000} & \multicolumn{4}{|c|}{ Week 18 fune-24 fune 1999} \\
\hline \multirow[b]{2}{*}{ Rates $\times 1000$} & \multicolumn{7}{|l|}{ Week } & \multicolumn{2}{|c|}{ Expected fluctuations } & \multicolumn{2}{|l|}{ Limits } \\
\hline & 1 & 2 & 3 & 4 & 5 & 6 & 7 & Average & \pm & Upper & Lower \\
\hline Watery diarrhoea & 5.9 & 8.9 & 8.8 & 4.1 & 5.6 & 3.3 & 7.7 & 6.8 & 3.7 & 10.5 & 3.1 \\
\hline Upper respiratory infections & 22.9 & 28.7 & 31.6 & 17.8 & 19.8 & 16.1 & 20.3 & 19.6 & 15.8 & 35.4 & 3.8 \\
\hline Scabies/Head lice & 2.6 & 2.1 & 3.3 & 0.8 & 0.9 & 0.7 & 1.4 & 2.4 & 1.7 & 4.1 & 0.7 \\
\hline Fever of unknown origin (FUO) & 1.7 & 1.7 & 3.8 & 0.5 & 1.5 & 0.2 & 1.5 & 2.2 & 2.3 & 4.6 & 0 \\
\hline
\end{tabular}




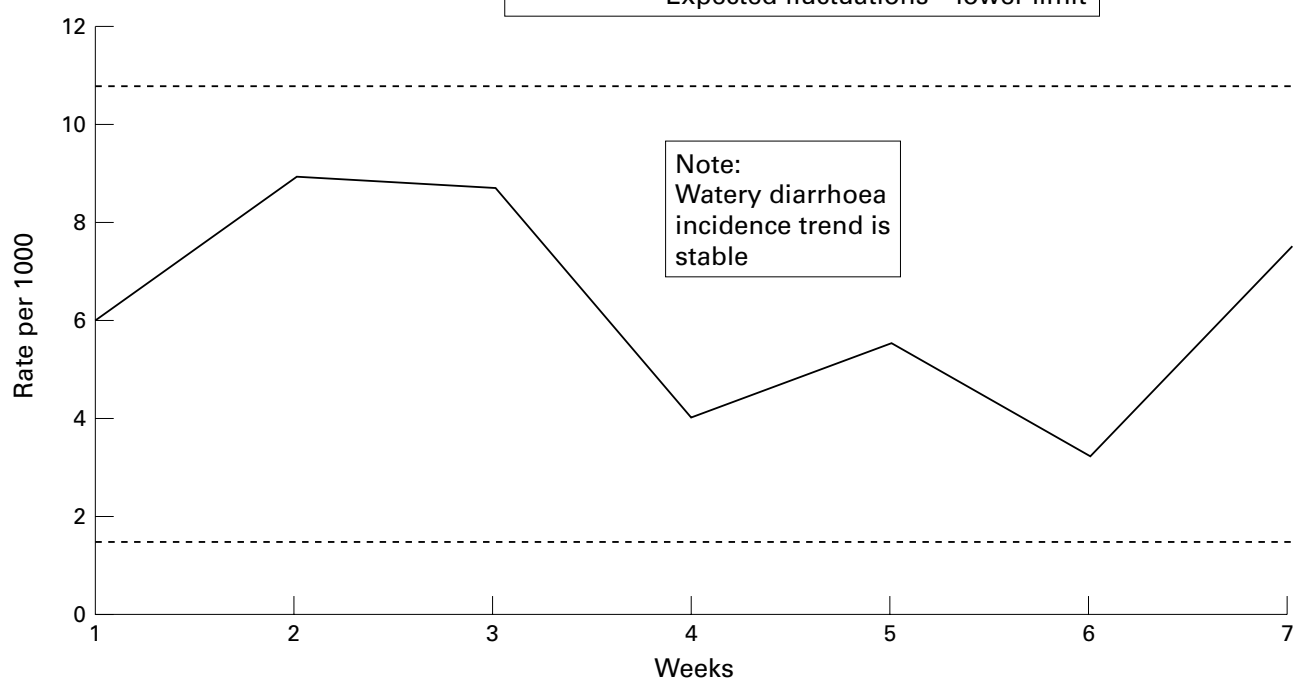

Figure 3 Example of the graph with the rate of reports of selected communicable disease per camp.

meaningful denominator on a weekly basis. Mortality data were calculated later on a three month time span.

Tuberculosis had a special register (WHO TB Register 03) with special feedback for the agencies authorised for treatment.

\section{Discussion}

The system has been developed through discussions and negotiations between all the parties involved and was of course limited by the scarce resources available at the time. It is a compromise between getting information that is reliable, but also rapid enough to act upon.

The decision to use reporting of syndromes was linked to the fact that we preferred a system that was very sensitive, even if not specific, because of the potentially explosive situation of an epidemic in a refugee camp where timely control measures are paramount. In this way, and with syndromic case definitions, a health worker with basic training can alert the centre to a potentially dangerous situation. False alarms do happen, but are far more easy to deal with than outbreaks recognised too late. The system has worked reasonably well for the period it has been in place providing usually two or three alarms per week (mostly bloody diarrhoea or acute jaundice) that were confirmed about $50 \%$ of the time. No outbreak of diseases with epidemic potential occurred during the whole period under surveillance (May to July 1999), although isolated cases of shigellosis, salmonellosis, hepatitis A and meningitis have been confirmed. No cases of measles or cholera have been confirmed in the reported period. Acute flaccid paralysis surveillance was conducted following the WHO guidelines for the eradication of poliomyelitis.

Some of the diseases in the weekly report form were there for another rationale. Watery diarrhoea among other things is a proxy for quality of drinking water, food hygiene and sewage disposal. Pneumonia was a concern because of the very cold conditions that the refugees had to endure in the first period of the crisis. Upper respiratory tract infections were one of the first reasons for consultation and a major issue in the media reporting, and so we preferred to have it under surveillance. Sexually transmitted diseases were a very sensitive issue and part of the broader sexual and reproductive health indicators. Fever of unknown origin is a useful indicator to detect infectious diseases missed by other syndromes and gives an idea of the request for health services by the population.

Scabies in the refugee situation is usually used as a proxy for access to water and head lice for crowding. The total number of consultations per week was used by some of the NGOs to calculate the burden of care and plan the opening times for their facilities. Referral to hospitals gave us some knowledge of the need for secondary care. Number of deaths, with sex, age and cause of deaths, together with number of births and stillbirths, are necessary to calculate the basic health indicators in a population.

\section{Conclusions}

During the period the surveillance system was in place (late April to August 1999) in the camps in fYROM no major outbreaks occurred and very few cases of diseases of epidemic potential were confirmed. Health needs assessments have been conducted both for the refugees in the camps and for the ones in host families confirming that communicable diseases have been contained to low levels during the crises compared with other massive refugee situations. There are various reasons for this. A population that started from a reasonably low level of prevalence of communicable diseases; a weather pattern that did not facilitate the spreading of gastroenteric diseases; a reasonably prompt response from the international community; and possibly a higher level of resources used per refugee compared with similar previous humanitarian crises. The 
surveillance system contributed to the situation, raising the alertness of health workers to infectious diseases and preparing the system for early action.

The most reported communicable diseases were urinary tract infections, particularly in children under 5 years of age, but the incidence diminished with the warmer weather. Despite the high rate of reporting these infections never raised to levels of major concern. With the coming of the summer watery diarrhoea increased, but always at acceptable levels. One of the major increases in diarrhoea was linked to consumption of undiluted fruit syrup that caused the symptom because of its high osmolarity. None of the other illness or syndromes reached alarming levels of reporting. Traumas and injuries were reported very rarely and in most of the cases were minor.

The surveillance system in the camps in fYROM was set up and maintained by the staff of the WHO Humanitarian Assistance Office in Skopje. Substantial help in its design and day to day functioning was received by WHO headquarters in Geneva, WHO-EURO in Copenhagen, the UNHCR health staff in Skopie, the fYROM Ministry of Health and all Governmental and pje, the fYROM Ministry of Health and all Governmental and
Non-Governmental Organisations providing health services in Non-Gover
the camps. 